This is the author's copy of the publication as archived in the DLR electronic library at http://elib.dlr.de. Please consult the original publication for citation, see https://arc.aiaa.org/doi/abs/10.2514/6.2022-1084.

\title{
Tilt-Wing Control Design for a Unified Control Concept
}

\author{
Daniel Milz and Gertjan Looye
}

Urban Air Mobility (UAM) promises an economic and ecological solution for the growing mobility demand by utilizing Electric Vertical Takeoff and Landing Vehicles (eVTOLs). Tilt-wing eVTOLs (e.g., Airbus A3 Vahana) appear to be the most promising ones because they offer an efficient wing-borne cruise flight while reducing the need for groundbased infrastructure at the cost of a complex control task. Tilt-wing vehicles increase the pilot's workload and introduce possible human and technical failures due to mechanical complexity. A unified control concept shall be able to handle the vehicle in every phase and provides a single clean and intuitive interface. This work develops a controller capable of decoupling the physical couplings of the flight dynamics. An integrated six-degree-of-freedom rigid body model in a compact mathematical representation is proposed, and flight control requirements are identified. An Incremental Nonlinear Dynamic Inversion (INDI) controller is designed which fulfills the requirements. Moreover, multiple command filters and outer-loop controllers are designed to handle different control modes and provide a proof-of-concept for a unified control scheme. Finally, the closed-loop system is evaluated by means of the control requirements and a generic UAM mission. The closed-loop system masters all parts of the mission and fulfills these requirements. The developed dynamic model and control system will be valuable for future tilt-wing eVTOL research, especially subsequent works on unified control systems.

\section{Copyright Notice}

Copyright (C) 2022 by German Aerospace Center (DLR). Published by the American Institute of Aeronautics and Astronautics, Inc., with permission.

Milz, Daniel and Looye, Gertjan (2022) "Tilt-Wing Control Design for a Unified Control Concept," AIAA 2022-1084. AIAA SCITECH 2022 Forum. January 2022. 


\title{
Tilt-Wing Control Design for a Unified Control Concept
}

\author{
Daniel Milz* and Gertjan Looye ${ }^{\dagger}$ \\ Institute of System Dynamics and Control, German Aerospace Center (DLR), 82234 Weßling, Germany
}

\begin{abstract}
Urban Air Mobility (UAM) promises an economic and ecological solution for the growing mobility demand by utilizing Electric Vertical Take-off and Landing Vehicles (eVTOLs). Tiltwing eVTOLs (e.g., Airbus A $^{3}$ Vahana) appear to be the most promising ones because they offer an efficient wing-borne cruise flight while reducing the need for ground-based infrastructure at the cost of a complex control task. Tilt-wing vehicles increase the pilot's workload and introduce possible human and technical failures due to mechanical complexity. A unified control concept shall be able to handle the vehicle in every phase and provides a single clean and intuitive interface. This work develops a controller capable of decoupling the physical couplings of the flight dynamics. An integrated six-degree-of-freedom rigid body model in a compact mathematical representation is proposed, and flight control requirements are identified. An Incremental Nonlinear Dynamic Inversion (INDI) controller is designed which fulfills the requirements. Moreover, multiple command filters and outer-loop controllers are designed to handle different control modes and provide a proof-of-concept for a unified control scheme. Finally, the closed-loop system is evaluated by means of the control requirements and a generic UAM mission. The closed-loop system masters all parts of the mission and fulfills these requirements. The developed dynamic model and control system will be valuable for future tilt-wing eVTOL research, especially subsequent works on unified control systems.
\end{abstract}

\section{Introduction}

IRCRAFT capable of vertical take-off and landing (VTOL) have been part of aeronautical research for decades [1]. A However, except for helicopters and some special, mostly military configurations, VTOL aircraft have not made it to the civil market yet [1]. Recent technological developments (e.g., distributed electric propulsion) and trends on novel mobility concepts, i.e., (On-Demand) Regional and Urban Air Mobility (UAM), led to a renaissance of VTOL vehicles in the form of electric vertical take-off and landing (eVTOL) vehicles [1]. There are various concepts for realizing eVTOLs [2]. The most common solution is the tilting propulsion system concept [3]. Compared to other VTOL systems, like multicopters, especially tilt-wing VTOL aircraft feature higher airspeeds and extended flight ranges in a trade-off with mechanical complexity and complex flight control systems [4].

One promising vehicle concept for research studies on tilt-wing eVTOL vehicles is the Airbus $A^{3}$ Vahana configuration. It has the advantage of an open-source design study [5, 6] and thus allows detailed analysis in research. Moreover, various research studies are based on this configuration, e.g., [7-79]. Additionally, the vehicle design consists of mostly known parts and does not require, for instance, little studied propulsion concepts. The used aircraft configuration is shown in [10] and consists of a fuselage and tandem tilt-wings with two canard wings in the front and two main wings in the back, each carrying two propeller engines and one control surface.

Tilt-wing aircraft already gained attention in the 1950s and 1960s through companies as Boeing, Ling-Temco-Vought (LTV), Hiller, and Canadair that developed flying tilt-wing prototype aircraft [11]. However, due to "challenges related to control and stability as well as mechanical complexity" [11], tilt-wing aircraft mostly vanished from active development and research. Current technological developments allow new ways to overcome these issues. Nevertheless, most of the current research is focused on small tilt-wing unmanned aerial vehicles (UAVs), dominated by tail-sitter UAVs, e.g., in [4, 12, 13]. Tilt-wing eVTOL control concepts for piloted Air Mobility solutions are only little studied so far.

The control design problem for tilt-wing eVTOL vehicles is a complex task. Hybrid aircraft, which can fly horizontally and vertically, handle the horizontal and vertical flight phases, as well as the transition, mostly separately in the control system [4]. However, this approach has notable drawbacks. First, the controller has to switch or schedule, which introduces possibly error-prone functionalities. Second, the input device (inceptor) mapping most likely changes during the different phases. This introduces a significant security risk since human errors are likely in such

\footnotetext{
*Researcher, daniel.milz@dlr.de Department of Aircraft System Dynamics, AIAA member

${ }^{\dagger}$ Head of Department, gertjan.looye@dlr.de Department of Aircraft System Dynamics, AIAA member
} 
a constellation [3]. Thus, intensive pilot training is required, which increases operational costs. Since many eVTOL concepts will be operated parallel, extra training and certification for every configuration are necessary. Even if the vehicle flies autonomously, the inner-loop controller takes different command variables for each phase. This behavior brings additional complexity and possible failures to all outer-loop and autopilot control systems.

One promising approach is to use the same inner-loop control system for all cases and provide control modes for different situations through outer-loop controllers and command filters, i.e., a unified control system [3]. However, in order to develop the aforementioned framework, a suitable inner-loop controller is required. The different control modes implement different operational modes via the same interface to the inner loop. Thus, it is advantageous if the inner loop is capable of decoupling the physical couplings. This would decrease the complexity of the control mode implementations. For instance, a vertical velocity control loop can utilize the vertical acceleration command channel of the vertical acceleration. A height controller can then be realized via an additional loop around the vertical velocity controller and the vertical acceleration command channel. Besides the decoupling capability, this controller should meet relevant flight control standards for tilt-wing eVTOLs in UAM operation.

\section{A. Related Work}

The concept of a unified control system probably first came up at the UK Royal Aircraft Establishment with the development of Vector thrust Aircraft Advanced Control (VAAC) for the AV-8B Harrier in the 1970s to 1980s [14]. The VAAC Harrier has sought a solution to the Harrier's "three-hand" problem where the pilot must simultaneously handle the thrust lever, stick, and the nozzle angle lever during take-off and landing. The VAAC Harrier was modified to be operated with a hand-on-throttle-and-stick control principle by pilots without special training [3, 15]. The research results were never actually implemented as another Harrier update. However, these results were fundamental for developing the F-35B Lightning II [14].

Lombaerts et al. make a step in developing a unified control system for eVTOL vehicles by reviewing the history and discussing the development and evaluation of simplified vehicle operation concepts using simplified models, controls, inceptors, and displays in [3]. According to the authors, these aspects are "inseparably interconnected" [3]. However, the publication's primary focus is not the inner-loop control system but the outer-loop command modes and envelope limitations. Nevertheless, the published results and learnings help design and optimize an outer-loop control system and the inceptor-command mappings.

In the publications [16, 17], the authors design an inner-loop control system for an eVTOL vehicle with separate horizontal and vertical propulsion systems. Their approaches use different forms of feedback-linearization control. The used control concepts, nonlinear dynamic inversion (NDI), and incremental nonlinear dynamic inversion (INDI) promise to control a plant in different regimes with different dynamics. They further focus on a unified command framework and describe their findings. A possible structure for a unified control system is introduced. The results are evaluated through current UAM scenarios. Both publications provide a valuable basis for this work. However, they do not consider tilting propulsion or tilt-wing concepts but a lift and cruise concept.

In 2016, Di Francesco and Mattei published their work on modeling a tilt-rotor vehicle and developing the corresponding control system based on INDI [18]. The authors conclude that the INDI approach was an "effective way to solve the problem of the dynamic inversion of systems non-affine in the control" [18].

In [13], Yanguo and Huanjin use an eigenstructure assignment algorithm to control a small tilt-rotor aircraft. However, this approach mainly depends on an adequately evaluated model, which is not available in this works' scope and is hard to realize in a practical implementation.

Hartmann et al. published various findings on tilt-wing aircraft. In [19], the authors attempt to control tilt-wing aircraft via the following control law: A predefined mapping between trim states and control outputs provides the trimmed control output. A disturbance realized via the inversion of a linear control effector matrix gives a mapping between desired forces and moments and control outputs. This disturbance can be used to accelerate or rotate the aircraft. However, this method requires the state to be in a "local attractor's sphere of influence" [19]. Large or fast disturbances thus lead to undetermined and possibly unstable behavior.

In [4], the authors show a method for a unified velocity control scheme for tilt-wing aircraft, improving the method from [19]. They introduced virtual control inputs to combine the effect of multiple control inputs. A control allocation scheme is, therefore, needed. The control allocation is a static mapping. Tilting the wing in "thrust-borne" [4] (vertical) flight or adjusting the body pitch angle in "wing-borne" [4] (horizontal) flight changes the effective wing angle. The primary control law uses gain scheduling: The control effectiveness matrix that maps control outputs to forces and moments is inverted. Thus desired forces and moments can be used to calculate corresponding control outputs. A "set 
of characteristic maps" [4] stores the inverse of the effectiveness matrix.

Another research group published several papers on the construction, aerodynamic analysis, and control design of a novel quad tilt-wing UAV [12, 20, 21]. In [12], they describe the process from the initial design, over the model generation and analysis, and the control design to experimental tests. The control system uses virtual control inputs from a high-level controller to a low-level controller scheduled depending on the current tilt angle via a lookup table [21]. The tilt angle follows the required velocity [21]. Although the published results are promising and describe the complete progress, the used tilt-wing aircraft is small and consists of only four propellers. Furthermore, the used control structure is not designed for large UAM applications but small radio-controlled (RC) vehicles.

\section{B. Approach}

This work proposes a unified control function for an integrated six-degree-of-freedom rigid body model based on [10]. Relevant flight control requirements for the unified control scheme (e.g., decoupling capabilities) and UAM operations and certification (e.g., according to air- and rotorcraft standards) are identified. A suitable flight control function is chosen and designed with the stated requirements in mind. Multiple command filters and flight control modes are implemented to cover different UAM-relevant flight phases, provide a proof-of-concept for the unified control concept, and complete a generic UAM mission. The closed-loop system is evaluated by means of the control requirements and the UAM mission.

\section{Integrated Model}

The tilt-wing eVTOL dynamic model is based on a simplified version of the model published in [10]. The basis of the integrated dynamic model consists of the 6-DOF rigid body flight mechanical equations of motion and the relevant forces and moments acting during the different flight phases, i.e., mainly propulsive and aerodynamic forces and moments.

Assuming a flat-earth environment, let ${ }^{\mathrm{N}} \mathbf{x} \in \mathbb{R}^{3}$ denote the aircraft's position vector in the north-east-down frame $\mathcal{F}_{\mathrm{N}},{ }^{\mathrm{B}} \mathbf{v}=\left[\begin{array}{lll}u & v & w\end{array}\right]^{T} \in \mathbb{R}^{3}$ the aircraft's velocity vector in body frame $\mathcal{F}_{\mathrm{B}},{ }^{\mathrm{N}} \boldsymbol{\Theta}=[\phi \theta \psi]^{T} \in[-\pi ; \pi]^{3} \subset \mathbb{R}^{3}$ the aircraft's rotation vector in $\mathcal{F}_{\mathrm{N}}$, and ${ }^{\mathrm{B}} \omega=\left[\begin{array}{ll}p & q\end{array}\right]^{T} \in \mathbb{R}^{3}$ the aircraft's angular velocity vector in $\mathcal{F}_{\mathrm{B}}$. Furthermore, let ${ }^{\mathrm{B}} \mathbf{f}_{\mathrm{a}} \in \mathbb{R}^{3}$ denote the total aerodynamic force, ${ }^{\mathrm{B}} \mathbf{f}_{\mathrm{p}} \in \mathbb{R}^{3}$ the total propulsive force, ${ }^{\mathrm{B}} \mathbf{f}_{\mathrm{g}}=m g[-\sin \theta \sin \phi \cos \theta \cos \phi \cos \theta]^{T} \in$ $\mathbb{R}^{3}$ the gravitational force, ${ }^{\mathrm{B}} \mathbf{m}_{\mathrm{a}} \in \mathbb{R}^{3}$ the total aerodynamic moment, and ${ }^{\mathrm{B}} \mathbf{m}_{\mathrm{p}} \in \mathbb{R}^{3}$ the total propulsive moment resolved in $\mathcal{F}_{\mathrm{B}}$. Then, the state space representation of the 6 degrees-of-freedom rigid-body flight mechanics are given as

$$
\left[\begin{array}{c}
{ }^{\mathrm{N}} \dot{\mathbf{x}} \\
{ }^{\mathrm{B}} \dot{\mathbf{v}} \\
{ }^{\mathrm{N}} \dot{\boldsymbol{\Theta}} \\
{ }^{\mathrm{B}} \dot{\omega}
\end{array}\right]=\left[\begin{array}{c}
{ }^{\mathrm{N}} \mathbf{R}_{\mathrm{B}}{ }^{\mathrm{B}} \mathbf{v} \\
-{ }^{\mathrm{B}} \omega \times{ }^{\mathrm{B}} \mathbf{v}+\frac{1}{m}{ }^{\mathrm{B}} \mathbf{f}_{g} \\
{ }^{\mathrm{N}} \mathbf{R}_{\mathrm{B}}{ }^{\mathrm{B}} \omega \\
-{ }^{\mathrm{B}} \omega \times\left(\mathbf{I}^{\mathrm{B}} \omega\right)
\end{array}\right]+\left[\begin{array}{c}
0 \\
\frac{1}{m}\left({ }^{\mathrm{B}} \mathbf{f}_{p}+{ }^{\mathrm{B}} \mathbf{f}_{a}\right) \\
0 \\
\mathbf{I}^{-1}\left({ }^{\mathrm{B}} \mathbf{m}_{a}+{ }^{\mathrm{B}} \mathbf{m}_{p}\right)
\end{array}\right]
$$

with the aircraft's mass $m=752.2 \mathrm{~kg}[8]$ and the components of the aircraft's inertia matrix I normalized with the aircraft's mass $m$ being $I_{\mathrm{xx}}=0.4183 \mathrm{~m}^{2}, I_{\mathrm{yy}}=1.8643 \mathrm{~m}^{2}, I_{\mathrm{zz}}=1.9484 \mathrm{~m}^{2}, I_{\mathrm{xy}}=0.2798 \mathrm{~m}^{2}, I_{\mathrm{xz}}=0 \mathrm{~m}^{2}$, and $I_{\mathrm{yz}}=0 \mathrm{~m}^{2}$. Additionally, ${ }^{\mathrm{N}} \mathbf{R}_{\mathrm{B}}$ represents the rotation matrix from frame $\mathcal{F}_{\mathrm{B}}$ into frame $\mathcal{F}_{\mathrm{N}}$. The propulsive forces and moments ${ }^{\mathrm{B}} \mathbf{f}_{\mathrm{p}}$ and ${ }^{\mathrm{B}} \mathbf{m}_{\mathrm{p}}$ are further defined as

$$
{ }^{\mathrm{B}} \mathbf{f}_{\mathrm{p}}=\left[\begin{array}{c}
\cos \left(\delta_{\text {tilt }}\right) \cdot T_{0} \\
0 \\
-\sin \left(\delta_{\text {tilt }}\right) \cdot T_{0}
\end{array}\right] \quad{ }^{\mathrm{B}} \mathbf{m}_{\mathrm{p}}=\left[\begin{array}{c}
\cos \left(\delta_{\text {tilt }}\right) \cdot \delta T_{\mathrm{pm}} \\
0 \\
-\sin \left(\delta_{\text {tilt }}\right) \cdot \delta T_{\mathrm{pm}}
\end{array}\right]+\left[\begin{array}{c}
r_{y} \sin \left(\delta_{\text {tilt }}\right) \cdot \delta T_{\mathrm{lr}} \\
\left(\cos \left(\delta_{\text {tilt }}\right) r_{z}+\sin \left(\delta_{\text {tilt }}\right) r_{x}\right) \cdot \delta T_{\mathrm{fr}} \\
r_{y} \cdot \cos \left(\delta_{\text {tilt }}\right) \cdot \delta T_{\text {lr }}
\end{array}\right]
$$

with the characteristic lengths $r_{\mathrm{x}}=3 \mathrm{~m}, r_{\mathrm{y}}=1 \mathrm{~m}$, and $r_{\mathrm{z}}=0.5 \mathrm{~m}$, the tilt angle $\delta_{\text {tilt }} \in\left[0 ; \frac{\pi}{2}\right]$, and the nominal thrust $T_{0} \in[0 \mathrm{~N} ; 1635 \mathrm{~N}]$. Furthermore, $\delta T_{\mathrm{pm}}$ denotes the torque difference between clockwise and counter-clockwise rotating rotors to zero, $\delta T_{\mathrm{fr}}$ the difference between front and rear rotors to zero, and $\delta T_{\mathrm{lr}}$ the difference between left and right rotors to zero. The aerodynamic forces and moments ${ }^{\mathrm{B}} \mathbf{f}_{a}$ and ${ }^{\mathrm{B}} \mathbf{m}_{a}$ are defined according to [10] as

$$
{ }^{\mathrm{B}} \mathbf{f}_{\mathrm{a}}=q S{ }^{\mathrm{B}} \mathbf{R}_{\mathrm{W}}\left[\begin{array}{c}
-C_{\mathrm{D}}\left(\alpha_{\mathrm{eff}}, \beta\right) \\
C_{\mathrm{S}}\left(\beta,{ }^{\mathrm{B}} \omega_{\mathrm{z}}\right) \\
-C_{\mathrm{L}}\left(\alpha_{\mathrm{eff}}\right)
\end{array}\right] \quad{ }^{\mathrm{B}} \mathbf{m}_{\mathrm{a}}=q S{ }^{\mathrm{B}} \mathbf{R}_{\mathrm{W}}\left[\begin{array}{c}
b C_{\mathrm{l}}\left(\beta,{ }^{\mathrm{B}} \omega_{\mathrm{x}},{ }^{\mathrm{B}} \omega_{\mathrm{z}}, \delta_{\mathrm{A}}\right) \\
c C_{\mathrm{m}}\left(\alpha_{\mathrm{eff}}{ }^{\mathrm{B}} \omega_{\mathrm{y}}, \delta_{\mathrm{E}}\right) \\
b C_{\mathrm{n}}\left(\beta,{ }^{\mathrm{B}} \omega_{\mathrm{x}},{ }^{\mathrm{B}} \omega_{\mathrm{z}}, \delta_{\mathrm{A}}\right)
\end{array}\right]
$$


with the rotation matrix ${ }^{\mathrm{B}} \mathbf{R}_{\mathrm{W}}$ from wind frame to body frame, the reference span width $b=6.87 \mathrm{~m}$, mean chord length $c=0.65 \mathrm{~m}$, and the reference wing area $S=8.93 \mathrm{~m}$, as well as the dynamic pressure $q=\frac{1}{2} \rho V^{2}$ with $\rho=1.225 \mathrm{~kg} \mathrm{~m}^{-3}$. The angle of attack $\alpha$, the sideslip angle $\beta$, and the airspeed $V$ are defined according to [10]. $\delta_{\mathrm{A}}$ and $\delta_{\mathrm{E}}$ are the aileron and elevator deflections, respectively. The effective angle of attack is $\alpha_{\text {eff }}=\alpha+\delta_{\text {tilt. }}$ The aerodynamic coefficients are approximated in [10].

The control input vector is $\mathbf{u}=\left[\delta_{\text {tilt }} T_{0} \delta T_{\mathrm{pm}} \delta T_{\mathrm{fr}} \delta T_{\mathrm{lr}} \delta_{\mathrm{A}} \delta_{\mathrm{E}}\right]^{T}$, the state vector is $\mathbf{x}=\left[{ }^{\mathrm{N}} \mathbf{x}{ }^{\mathrm{B}} \mathbf{v}^{\mathrm{N}} \boldsymbol{\Theta}{ }^{\mathrm{B}} \omega\right]^{T}$, and the output vector of the full-state observable system is $\mathbf{y}=\left[{ }^{\mathrm{N}} \mathbf{x}^{\mathrm{B}} \mathbf{v}^{\mathrm{N}} \boldsymbol{\Theta}{ }^{\mathrm{B}} \omega \alpha \beta V\right]^{T}$. The control inputs follow a first-order low-pass model

$$
H(s)=\frac{1}{\tau s+1}
$$

where $\tau$ denotes the time constant of the filter. Thus, the commanded control input is $\mathbf{u}_{\mathrm{c}}$ with $\mathbf{u}=H(s) \mathbf{u}_{\mathrm{c}}$. The time constants differ between the inputs. The motor time constants are assumed to be $\tau_{T_{0}}=\tau_{\delta T_{\mathrm{pm}}}=\tau_{\delta T_{\mathrm{fr}}}=\tau_{\delta T_{\mathrm{Ir}}}=24 \mathrm{~ms}$ [22]. The aileron and elevator time constants are approximated by the values of a comparable-size aircraft, the Cessna 182, to be $\tau_{\delta_{\mathrm{A}}}=\tau_{\delta_{\mathrm{E}}}=60 \mathrm{~ms}[23]$. The tilt mechanism is approximated by $\tau_{\delta_{\text {tilt }}}=250 \mathrm{~ms}$ and $\left|\dot{\delta}_{\text {tilt }}\right| \leq 12^{\circ} \mathrm{s}^{-1}$, according to [24]. The maximum thrust value of $1650 \mathrm{~N}$ is chosen so that possible safety requirements are fulfilled including the ability to land safely in the case of two failed engines.

A trim point analysis of the open-loop model is shown in [10].

\section{Flight Control Requirements}

As a next step, the control system requirements are defined since a requirements-based control design approach is used.

\section{A. Unified Control Inputs}

In order to design a controller for a unified control concept, an overview of a possible unified control concept and its control input mappings is helpful. The main goal of a unified control system is to have a single inner-loop controller capable of handling all occurring flight phases, i.e., hover, transition, and cruise, while maintaining the same control inputs and the same control structure. Having the same control inputs in every phase reduces the complexity for the pilot and the outer-loop control system, reducing possible errors [3]. However, due to the vast difference between the flight phases, a blending of some control inputs may be unavoidable. In [3], the authors proposed multiple inceptor-command mappings. Two promising mappings there are "unified control" and "E-Z-fly". This unified control mapping is mainly focused on a flight-physical representation, which "brings the concept closer to what the pilot expects" [3]. The other promising mapping is based on the E-Z-Fly concept from [25]. This concept completely decouples the different axes making it "easy to fly" for untrained persons [25]. This E-Z-fly concept is focused on simplicity and avoiding control input changes while decoupling flight-physical relations.

Possible inceptor constellations consist of a 3-axis side stick and a lever. The presence of a side stick and a lever is reminiscent of a classic cockpit. Let $\delta_{\text {lat }}$ denote the sidestick's lateral axis, $\delta_{\text {lon }}$ its longitudinal axis, and $\delta_{\text {yaw }}$ its rotational yaw axis. The lever is denoted as $\delta_{\text {lever }}$. The three-axis sidestick commands horizontal movement and rotation. The forward movement is controlled by $\delta_{\text {lon }}$, the sidewards movement by $\delta_{\text {lat }}$, and the heading by $\delta_{\text {yaw }}$. The lever $\delta_{\text {lever }}$ commands the vertical movement, i.e., the height change $\dot{h}$.

The different mappings are shown in Table 1 , where $V_{\text {gnd }}$ denotes the absolute velocity over ground and $V_{\text {cas }}$ the calculated airspeed.

Table 1 Possible assignment of inceptors and control inputs during the different flight phases.

\begin{tabular}{|c|c|c|c|c|c|c|c|c|}
\hline & \multicolumn{4}{|c|}{ Unified control [3] } & \multicolumn{4}{|c|}{ E-Z-Fly [3] } \\
\hline & $\delta_{\text {lon }}$ & $\delta_{\text {lat }}$ & $\delta_{\text {yaw }}$ & $\delta_{\text {lever }}$ & $\delta_{\text {lon }}$ & $\delta_{\text {lat }}$ & $\delta_{\text {yaw }}$ & $\delta_{\text {lever }}$ \\
\hline Hover & $\dot{\mathbf{v}}_{\mathrm{x}}$ & $\mathbf{v}_{\mathrm{y}}$ & $\dot{\psi}$ & $\dot{h}$ & $\dot{\mathbf{v}}_{\mathrm{x}}$ & $\mathbf{v}_{\mathrm{y}}$ & $\dot{\psi}$ & $\dot{h}$ \\
\hline Transition & $V_{\text {gnd }}$ & $\phi$ & $\beta$ & $\gamma$ or $\dot{h}$ & $\dot{V}_{\text {gnd }}$ & $\mathbf{v}_{\mathrm{y}}$ & $\dot{\psi}$ & $\dot{h}$ \\
\hline Forward flight & $V_{\text {cas }}$ & & $\beta$ & $\gamma$ or $\dot{h}$ & $\dot{V}_{\text {cas }}$ & $\mathbf{v}_{\mathrm{y}}$ & $\dot{\psi}$ & $\dot{h}$ \\
\hline
\end{tabular}


This development of a unified control concept is beyond the scope of this work. It may be the focus of future research and studies.

\section{B. Control Requirements}

The controller is designed based on certain handling quality requirements. Various standards propose means of assessment for aerospace vehicles. However, no dedicated standard has been published for eVTOL vehicles and their application in UAM [26]. Thus, a selection of handling quality standards for rotorcraft (ADS-33E-PRF [27]) and aircraft (AS94900A [28] and MIL-HDBK 1797 [29]) is used. Note that other authors use the same approach, e.g., [17, 26, 30]. The requirements ultimately applied are listed below.

Damping ratio and stability margins A damping ratio of $\zeta \geq 0.35$ is required in the "hover and low speed requirements" in [27]. However, in [27], no stability margins are directly defined. Thus, as done by [26], the stability margin requirements are obtained from AS94900. The gain margin has to be $\geq 6 \mathrm{~dB}$ while maintaining a phase margin of $\geq 45^{\circ}$.

Height response characteristics The height response characteristic in hover mode, measured via a step input on the lever, shall be in the form of a first-order response [27, 3.3.10.1]. More specifically, "the vertical rate response shall have a qualitative first-order appearance for at least 5 seconds following a step collective input" [27, 3.3.10.1]. The attitude shall be maintained essentially constant. Thus, the response shall be similar to the following transfer function in the Laplace domain

$$
\frac{\dot{h}}{\delta_{\text {lever }}}=\frac{K}{T_{\mathrm{h}} s+1} \cdot \exp \left(-\tau_{\mathrm{h}} s\right)
$$

where $\tau_{\mathrm{h}}$ characterizes the time delay and $T_{\mathrm{h}}$ the time constant. The closed-loop system requires $T_{\mathrm{h}} \leq 5 \mathrm{~s}$ and $\tau_{\mathrm{h}} \leq 0.2 \mathrm{~s}$. The values are obtained by time-domain fitting where the coefficient of determination $r^{2}$ has to be $0.97<r^{2}<1.03[27$, 3.3.10.1].

Interaxis coupling ADS-33E-PRF states [27, 3.3.9, 3.4.5] that the control inputs to achieve a response in one axis shall not result in objectionable responses in any other axis, where the most relevant ones are [27, 3.3.9.2, 3.4.5.1, 3.4.5.2]. Let $\Delta \phi_{4}$ and $\Delta \theta_{4}$ denote the deviation from trim at end of a 4 seconds step period on the roll angle $\phi$ and the pitch angle $\theta$ respectively. Let $\Delta \theta_{\mathrm{pk}}, \Delta \phi_{\mathrm{pk}}$, and $\Delta n_{\mathrm{z}_{\mathrm{pk}}}$ denote peak deviation of the corresponding quantity within the step period. Then, a 4 seconds step input on $\phi$ may only exhibit a disturbance in the pitch axis of $-\frac{1}{4} \leq \frac{\Delta \theta_{\mathrm{pk}}}{\Delta \phi_{4}} \leq \frac{1}{4}$. Vice versa, a 4 seconds step input on $\theta$ may only exhibit a disturbance in the roll axis of $-\frac{1}{4} \leq \frac{\Delta \phi_{\mathrm{pk}}}{\Delta \theta_{4}} \leq \frac{1}{4}$. Furthermore, the peak pitch angle deviation shall be small during a step on the vertical vertical acceleration $n_{\mathrm{z}}$ caused by a change on the collective and thus motor torque $Q$. For a small change of the motor torque of $\Delta Q<20 \%,\left|\frac{\Delta \theta_{\mathrm{pk}}}{\Delta n_{\mathrm{pk}}}\right| \leq 0.0573 \frac{{ }^{\circ} \mathrm{s}^{2}}{\mathrm{~m}}$. However, for large changes on the motor torque of $\Delta Q \geq 20 \%,\left|\frac{\Delta \theta_{\mathrm{pk}}}{\Delta n_{\mathrm{p}_{\mathrm{pk}}}}\right| \leq 0.0286 \frac{\mathrm{o}^{2}}{\mathrm{~m}}$ shall hold for an upwards acceleration and $\left|\frac{\Delta \theta_{\mathrm{pk}}}{\Delta n_{z_{\mathrm{pk}}}}\right| \leq 0.0143 \frac{{ }^{\circ} \mathrm{s}^{2}}{\mathrm{~m}}$ for a downwards acceleration.

Translational rate response The translational rate response describes the ability of rotorcraft to accelerate horizontally by changing the roll or pitch angle. ADS-33E-PRF requires this translational rate response to fulfill [27, 3.3.12]:

For Response-Types designated as Translational Rate Command, the translational rate response to step cockpit pitch (roll) control position or force inputs shall have a qualitative first order appearance, and shall have an equivalent rise time, $T_{\mathrm{x}}\left(T_{\mathrm{y}}\right)$, no less than 2.5 seconds and no greater than 5 seconds. [ ...] the following requirements shall apply:

- The pitch and roll attitudes shall not exhibit objectionable overshoots in response to a step

- Zero cockpit control force and deflection shall correspond to zero translational rate with respect to fixed objects, or to the landing point on a moving ship.

- There shall be no noticeable overshoots in the response of translational rate to control inputs. The gradient of translational rate with control input shall be smooth and continuous. 


\section{Mission Definition}

Uber has done much conceptual research in UAM and defined a generic mission trajectory [31]. A conceptually derived mission trajectory is shown in Figure 1

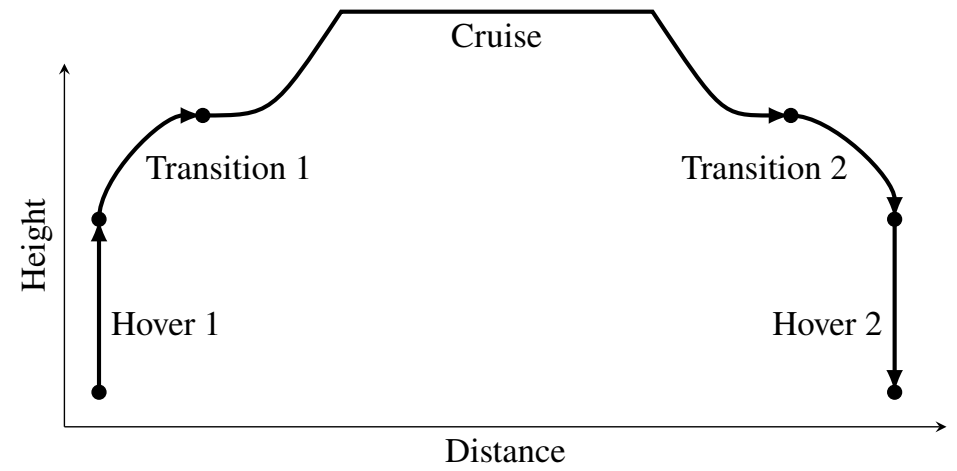

Fig. 1 Generic mission trajectory from [31].

\section{Control Theory}

Section I.A includes related work suggesting promising control law candidates, including gain scheduling control laws, eigenstructure assignment, nonlinear dynamic inversion (NDI), and incremental nonlinear dynamic inversion (INDI). Inversion-based control laws show significant advantages in flight control, especially when aiming to decouple the axes and thus the basis for a unified inner-loop [16-18]. An outer-loop linear controller combined with a reference model can be used to set and tune the dynamic behavior to ensure handling qualities [32]. Due to the axes decoupling, the outer-loop construct does not directly depend on the system's physical coupling. The axes and different states can be controlled directly and independently. This property allows providing a single interface.

A common inversion-based control law is feedback linearization or NDI. Those control laws depend heavily on the knowledge of the internal dynamics. Due to complex and partly unknown aerodynamic effects, especially effects occurring during the transition or caused by propeller-wing or propeller-propeller interactions, the internal dynamics are not fully known [10]. Thus, an adaptive or robust control law that ideally does not depend on the knowledge of the internal dynamics is necessary.

Recent developments yielded the INDI control law [33-38]. This incremental version of NDI substitutes internal dynamics with measurements. The measurements of the state derivatives, i.e., the acceleration and angular acceleration, however, have to be synchronized and must have a sufficient sampling frequency [37]. Future eVTOL vehicles strive for autonomy, where plenty of equipped sensors are necessary and thus likely fulfill the requirements of the INDI control law.

Assume a control affine explicit state-space system with the dynamics $\dot{\mathbf{x}}=\mathbf{f}(\mathbf{x})+\mathbf{G}(\mathbf{x}) \mathbf{u}$ and a relative degree of 1 . Then, the INDI law can be stated as [39]

$$
\mathbf{u}=\mathbf{G}\left(x_{\mathrm{m}}\right)^{-1}\left(v-\dot{\mathbf{x}}_{\mathrm{m}}+\mathbf{G}\left(x_{\mathrm{m}}\right) \mathbf{u}_{\mathrm{m}}\right)
$$

where $\dot{\mathbf{x}}_{\mathrm{m}}$ is the measured state derivative, $\mathbf{u}_{\mathrm{m}}$ the measured control input, and $v$ the pseudo control input. In [39], the author shows that (6) is an advantageous formulation of the INDI law for control allocation.

\section{Controller Design and Integration}

This section describes the implementation of an INDI controller for a unified control scheme according to the requirements from Section III.B. The implementation contains the realization of (6) and the control structure with filters and linear outer-loop controllers. The inner loop handles all flight phases, whereas the outer-loop controllers allow different flight control modes. In [37] and [3, 16, 17], the authors implement a similar control law and develop special functions for the outer-loop. A parameter tuning and optimization is performed for all cases where the initial settings are insufficient. Multi-objective optimization of the horizontal velocities' step response is performed. 


\section{A. Controller Structure}

The principal structure of the controller is shown in Figure 2 which is a common structure in flight control design with inversion based methods [17, 32, 37]. The controller consists of the following parts:

- INDI core and control allocation: Actual inversion of the required accelerations or forces and moments.

- Linear controllers: Linear controllers around the inversion.

- Reference model: A reference model of $n^{\text {th }}$ order to filter the command

- Command filtering: Implementation of different flight modes.

- Autopilot: Implementation of auto-flight functions and trajectory planning.

- Pilot input: Input device and filtering for cockpit inputs.

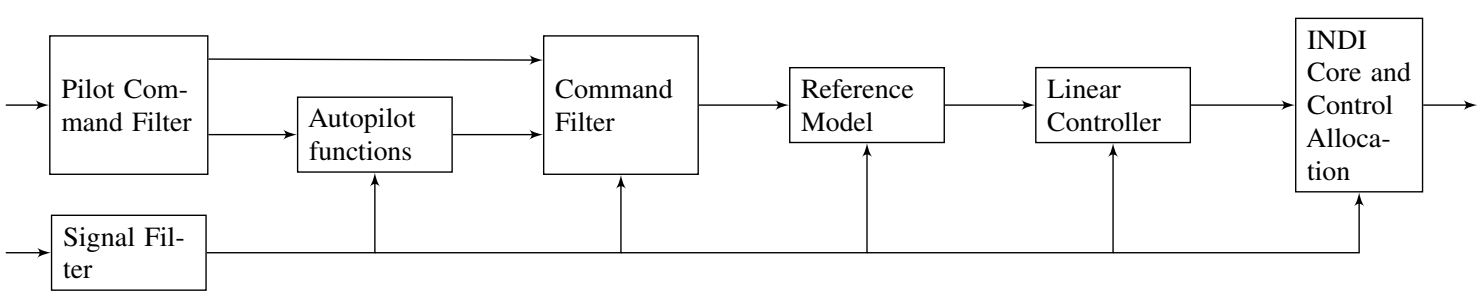

Fig. 2 Basic cascade structure of the implemented controller.

\section{B. INDI Control Implementation}

The controller implementation is based on the model (1). Note that the inputs $\delta T_{\mathrm{pm}}$ and $\delta_{\mathrm{A}}$, as well as $\delta T_{\mathrm{lr}}$ and $\delta_{\mathrm{E}}$, are redundant. Those inputs affect a change in the same state derivative. However, it holds that $\operatorname{rank} \mathbf{G}\left(x_{\mathrm{m}}\right) \leq 5$ for all possible $x_{\mathrm{m}}$, but $\mathbf{u} \in \mathbb{R}^{7}$. Thus, control allocation strategies are required to achieve the control law (6), e.g., [40]. In the scope of this work $\delta_{\mathrm{A}}=0$ and $\delta_{\mathrm{E}}=0$ holds to make $\mathbf{G}\left(x_{\mathrm{m}}\right)$ invertible. Note that, however, an optimization-based approach would most likely lead to superior results. The directly controllable or external states $\xi$ are ${ }^{\mathrm{B}} \mathbf{v}_{\mathrm{x}},{ }^{\mathrm{B}} \mathbf{v}_{\mathrm{z}},{ }^{\mathrm{B}} \omega_{\mathrm{x}}$, ${ }^{\mathrm{B}} \omega_{\mathrm{y}}$, and ${ }^{\mathrm{B}} \omega_{\mathrm{z}}$. The system of the external dynamics is

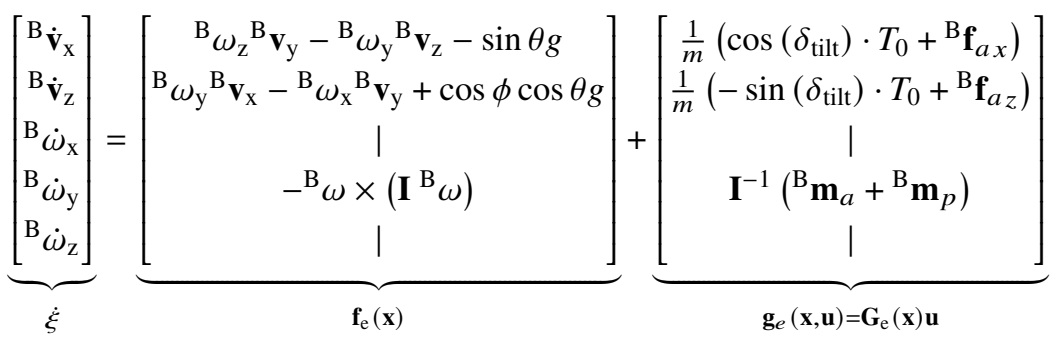

The control law (6) can thus be written as

$$
\mathbf{u}_{\mathrm{c}}=\mathbf{G}_{\mathrm{e}}^{-1}\left(\mathbf{x}_{\mathrm{m}}\right)\left(v-\xi_{\mathrm{m}}+\mathbf{g}_{\mathrm{e}}\left(\mathbf{x}_{\mathrm{m}}, \mathbf{u}_{\mathrm{m}}\right)\right)
$$

where $\xi_{\mathrm{m}}$ denotes the measured external states, $\mathbf{G}_{\mathrm{e}}^{-1}$ denotes the matrix inverse of $\mathbf{G}_{\mathrm{e}}, v$ the virtual control input, and $\mathbf{u}_{\mathrm{c}}$ finally the commanded system input. The index index $\mathrm{m}_{\mathrm{m}}$ denotes measured quantities. Note that the internal states, i.e., ${ }^{\mathrm{N}} \mathbf{x},{ }^{\mathrm{N}} \boldsymbol{\Theta}$, and ${ }^{\mathrm{B}} \mathbf{v}_{\mathrm{y}}$, remain and are not covered by the inversion. Since external states directly influence the internal states and no zero dynamics are present, the internal states are controlled by the outer-loop controller that stabilizes these states. Thus, it is sufficient to control the external states in order to control the system.

\section{Linear Controllers and Reference Models}

The linear outer-loop controller compensates for errors in the velocities and attitudes and commands the virtual control input $v$ to track the reference $x_{\text {ref }}$. The controller is realized in a PID-like way. A feed-forward path of the second derivative reference-filtered signal to the output is used [17, 37]. The error $x_{\mathrm{ref}}-x_{\mathrm{m}}$ between the reference $x_{\mathrm{ref}}$ and the measurement $x_{\mathrm{m}}$ is controlled via a PI controller with the gains $K_{\mathrm{P}}$ and $K_{\mathrm{I}}$. The error in the derivative, i.e., $\dot{x}_{\text {ref }}-\dot{x}_{\mathrm{m}}$, is controlled via a gain $K_{\mathrm{D}}$. $\ddot{x}_{\text {ref }}$ is en-/disabled by $K_{\mathrm{FF}} \in\{0 ; 1\}$ and represents the feed-forward path. The linear controller 
can be expressed as

$$
v=\left(K_{\mathrm{P}}+\frac{K_{\mathrm{I}}}{s}\right)\left(x_{\mathrm{ref}}-x_{\mathrm{m}}\right)+K_{\mathrm{D}}\left(\dot{x}_{\mathrm{ref}}-\dot{x}_{\mathrm{m}}\right)+K_{\mathrm{FF}} \ddot{x}_{\mathrm{ref}}
$$

with the parameter values given in Table 2 .

The reference signals are calculated via a reference model that takes the desired signal and optionally the derivative and outputs the filtered quantity and corresponding filtered first and second derivative. The commanded or desired signals $x_{\mathrm{d}}$, and its derivative $\dot{x}_{\mathrm{d}}$ are filtered via the second-order low-pass filter:

$$
H_{\text {ref }}(s)=\frac{\omega_{0}^{2}}{s^{2}+2 \zeta \omega_{0} s+\omega_{0}^{2}}
$$

with the natural frequency $\omega_{0}$ and damping ratio $\zeta$. The corresponding numerical values for the reference models of the different axes are given in Table 3 This filter outputs the filtered signal $x_{\text {ref }}$, its first derivative $\dot{x}_{\text {ref }}$, and its second derivative $\ddot{x}_{\text {ref }}$. The estimated and filtered outputs are sufficiently free of noise [37]. Thus, noise propagation into the control laws is avoided [37]. The filter in (10) is implemented, as shown in [17]. It is essential to initialize the integrators with the current measurement of the quantity $x_{\mathrm{m}}$ for the second integrator and the current measured derivative of the quantity $\dot{x}_{\mathrm{m}}$ for the first integrator.

\begin{tabular}{|c|c|c|c|c|c|c|c|c|c|}
\hline Parameter & Value & Parameter & Value & Parameter & Value & Parameter & Value & Parameter & Value \\
\hline$K_{\mathrm{P}, \mathrm{V}}$ & 2 & $K_{\mathrm{P}, \mathrm{h}}$ & 1 & $K_{\mathrm{P}, \phi}$ & 4 & $K_{\mathrm{P}, \theta}$ & 2 & $K_{\mathrm{P}, \psi}$ & 1 \\
\hline$K_{\mathrm{I}, \mathrm{V}}$ & 0 & $K_{\mathrm{I}, \mathrm{h}}$ & 0 & $K_{\mathrm{I}, \phi}$ & 0 & $K_{\mathrm{I}, \theta}$ & 0 & $K_{\mathrm{I}, \psi}$ & 0 \\
\hline \multirow[t]{2}{*}{$K_{\mathrm{FF}^{\prime}, \mathrm{V}}$} & 1 & $K_{\mathrm{D}, \mathrm{h}}$ & 10 & $K_{\mathrm{D}, \phi}$ & 8 & $K_{\mathrm{D}, \theta}$ & 4 & $K_{\mathrm{D}, \psi}$ & 2 \\
\hline & & $K_{\mathrm{FF}, \mathrm{h}}$ & 1 & $K_{\mathrm{FF}, \phi}$ & 1 & $K_{\mathrm{FF}, \theta}$ & 1 & $K_{\mathrm{FF}, \psi}$ & 1 \\
\hline
\end{tabular}

Table 2 Parameter values of the linear controller.

\begin{tabular}{|c|c|c|c|c|c|c|c|c|c|}
\hline Parameter & Value & Parameter & Value & Parameter & Value & Parameter & Value & Parameter & Value \\
\hline$\omega_{0, \mathrm{~V}}$ & 2 & $\omega_{0, \mathrm{~h}}$ & 2 & $\omega_{0, \phi}$ & 2 & $\omega_{0, \theta}$ & 0.5 & $\omega_{0, \psi}$ & 1 \\
\hline$\zeta_{\mathrm{V}}$ & 1 & $\zeta_{\mathrm{h}}$ & 0.8 & $\zeta_{\phi}$ & 1 & $\zeta_{\theta}$ & 1 & $\zeta_{\psi}$ & 1 \\
\hline
\end{tabular}

Table 3 Parameter values of the reference model.

\section{Command Filters}

Command filtering realizes different flight control modes [17]. The command filtering is separated into command filters for velocity, altitude, roll attitude, pitch attitude, and heading. They have different modes of control. The command filters are inspired by [17] and [37].

Altitude command filter The altitude command filter creates height commands $h_{\mathrm{c}}$ and its derivative $\dot{h}_{\mathrm{c}}$. For altitude control, three modes are available. The altitude can either be commanded directly, i.e., height command height hold $(\mathrm{HCHH})$, via its rate, i.e., rate command height hold $(\mathrm{RCHH})$, or via a mode holding the current altitude, i.e., no command height hold (NCHH). The altitude command filter can be described via Figure 3 with $K_{\text {eff, lever }}=2$.

The enable block $1 / 0$ represents the behavior where the derivative is passed through if the limited integrator is not saturated. If the limited integrator is saturated, the derivative signal will not be passed through. This construction is equivalent to a derivative of the limited integrator's output. The hold block is enabled when activating the NCHH mode. The block then stores the last height that passed through.

Translational rate command (TRC) The TRC command filter realizes the translational movement in horizontal directions of the vehicle in hover mode via rotations of the vehicle and thus its thrust vector [17]. The TRC is 


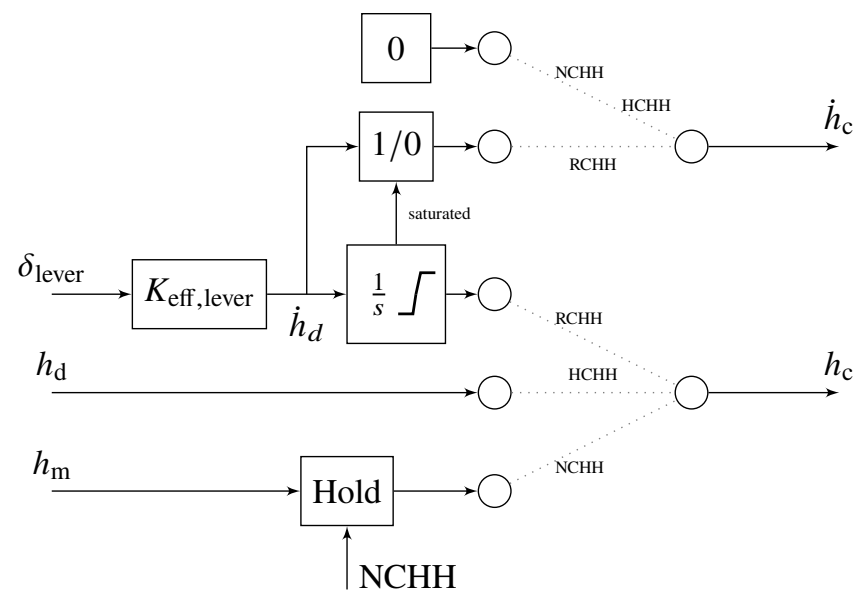

Fig. 3 Block diagram of the altitude command filter.

an inversion-based controller that tracks ground-based velocities in horizontal directions, i.e., ${ }^{\mathrm{N}} \dot{\mathbf{x}}_{\mathrm{x}}$ and ${ }^{\mathrm{N}} \dot{\mathbf{x}}_{\mathrm{y}}$. The implementation of the TRC is shown in Figure 4. This block diagram consists of a first-order low pass filter acting as a reference model, a linear controller, and a dynamic inversion block.

The TRC mode comes from rotorcraft operation and may be advantageous for eVTOL vehicles when operating in hover mode close to the ground. Possible maneuvers are take-off, landing, and taxiing. Especially when landing, precise maneuvering w.r.t. the ground is required.

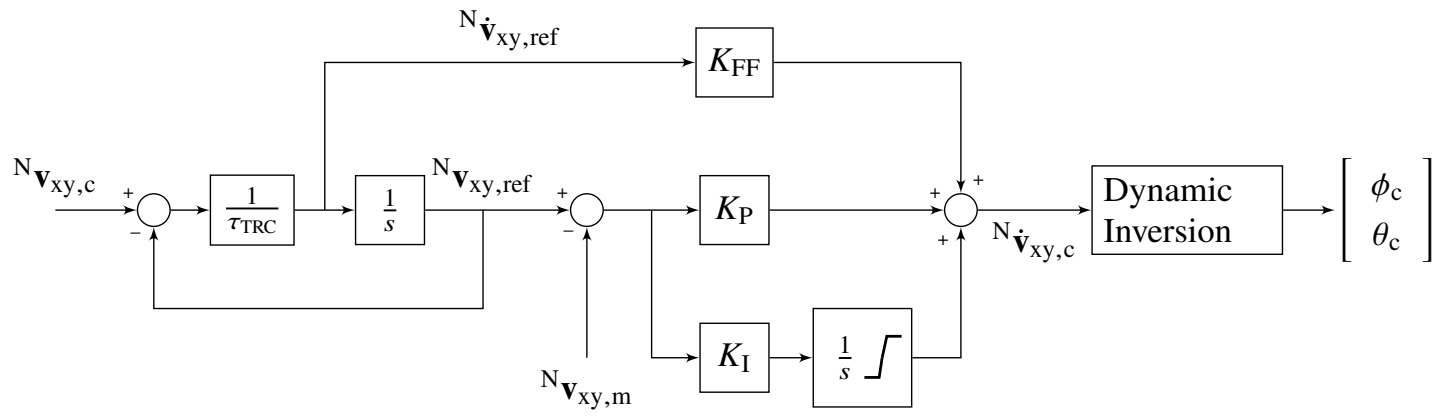

Fig. 4 Block diagram of the translational rate command filter.

The TRC filter can be expressed as

$$
{ }^{\mathrm{N}} \dot{\mathbf{v}}_{\mathrm{xy}, \mathrm{c}}=\left(K_{\mathrm{P}}+\frac{K_{\mathrm{I}}}{s}\right)\left({\frac{1}{\tau_{\mathrm{TRC}} s+1}}^{\mathrm{N}} \mathbf{v}_{\mathrm{xy}, \mathrm{d}}-{ }^{\mathrm{N}} \mathbf{v}_{\mathrm{xy}, \mathrm{m}}\right)+K_{\mathrm{FF}} \frac{s}{\tau_{\mathrm{TRC}} s+1}{ }^{\mathrm{N}} \mathbf{v}_{\mathrm{xy}, \mathrm{d}}
$$

The time constant $\tau_{\mathrm{TRC}}=2.5 \mathrm{~s}$ is chosen according to ADS-33E-PRF requirements [27]. The dynamic inversion can be approximated as

$$
\begin{aligned}
& \phi_{\mathrm{c}}=\arcsin \left(\frac{{ }^{\mathrm{N}} \dot{\mathbf{v}}_{\mathrm{x}, \mathrm{c}} \sin \psi+{ }^{\mathrm{N}} \dot{\mathbf{v}}_{\mathrm{y}, \mathrm{c}} \cos \psi}{\sqrt{\mathrm{N}_{\mathbf{v}_{\mathrm{x}, \mathrm{c}}^{2}}^{2}+{ }^{\mathrm{N}} \dot{\mathbf{v}}_{\mathrm{y}, \mathrm{c}}^{2}+\left({ }^{\mathrm{N}} \dot{\mathbf{v}}_{\mathrm{z}, \mathrm{c}}-g\right)^{2}} \sin \delta_{\text {tilt }}}\right) \\
& \theta_{\mathrm{c}}=\arctan \left(\frac{\left({ }^{\mathrm{N}} \dot{\mathbf{v}}_{\mathrm{x}, \mathrm{c}} \cos \psi-{ }^{\mathrm{N}} \dot{\mathbf{v}}_{\mathrm{y}, \mathrm{c}} \sin \psi\right) \cdot \cos \phi \tan \delta_{\text {tilt }}+{ }^{\mathrm{N}} \dot{\mathbf{v}}_{\mathrm{z}, \mathrm{c}}-g}{{ }^{\mathrm{N}} \dot{\mathbf{v}}_{\mathrm{x}, \mathrm{c}} \cos \psi-{ }^{\mathrm{N}} \dot{\mathbf{v}}_{\mathrm{y}, \mathrm{c}} \sin \psi+\left({ }^{\mathrm{N}} \dot{\mathbf{v}}_{\mathrm{z}, \mathrm{c}}-g\right) \cos \psi \tan \delta_{\text {tilt }}}\right)
\end{aligned}
$$

with the gravitational acceleration constant $g$. The linear controller consists of a feed-forward path with the gain $K_{\mathrm{FF}}=1$. The PI controller realized by the gains $K_{\mathrm{P}}=0.35$ and $K_{\mathrm{I}}=0.04$ stabilizes the error, i.e., ${ }^{\mathrm{N}} \mathbf{v}_{\mathrm{xy}}$, ref $-{ }^{\mathrm{N}} \mathbf{v}_{\mathrm{xy}, \mathrm{m}}$. While the 
inversion does not entirely reflect all occurring dynamics, the integrator handles those. For instance, the aerodynamic drag effects are not covered in the dynamics. Thus, a particular rotation is required to compensate for the drag in order to keep a commanded horizontal velocity. By adding another control cascade around the velocity controller, i.e., a proportional gain $K_{\mathrm{P}}=0.23$ on the position error, position control capability of the TRC can be achieved.

Roll command filter The roll command filter is similar to the altitude command filter in Figure 3 The roll angle command $\phi_{\mathrm{c}}$ and the roll angle derivative command $\dot{\phi}_{\mathrm{c}}$ depend on the selected mode, i.e., attitude command attitide hold (ACAH) or rate command attitude hold (RCAH). The roll command filter can be expressed as

$$
\phi_{\mathrm{c}}=\left\{\begin{array}{ll}
K_{\mathrm{eff}, \phi} \delta_{\text {lat }}, & \text { ACAH } \\
\frac{1}{s} K_{\mathrm{eff}, \phi} \delta_{\text {lat }}, & \text { RCAH }
\end{array} \quad \dot{\phi}_{\mathrm{c}}= \begin{cases}0, & \mathrm{ACAH} \\
K_{\mathrm{eff}, \dot{\phi}} \delta_{\text {lat }}, & \mathrm{RCAH}\end{cases}\right.
$$

Note that the integrator is realized by a limited integrator, which stops $\phi_{\mathrm{c}}$ when saturates. The minimum and maximum roll angle is set to $\pm \frac{\pi}{6}$. The effectiveness gains are $K_{\mathrm{eff}, \phi}=\frac{\pi}{6}$ and $K_{\mathrm{eff}, \dot{\phi}}=0.15$.

Pitch command filter The pitch command filter is similar to the roll command filter with the effectiveness gains $K_{\mathrm{eff}, \theta}=0.3$ and $K_{\mathrm{eff}, \dot{\theta}}=0.15$.

Velocity command filter The velocity command filter has a basic structure similar to the altitude command filter. Possible modes are velocity command velocity hold (VCVH) and rate command velocity hold (RCVH). The normalized velocity and acceleration stick command are transformed with a piecewise linear function, i.e., the effectiveness is represented via a piecewise linear weighting with $K_{\mathrm{eff}, \dot{V}}=0.1$

$$
K_{\text {eff }, \mathrm{V}}\left(\delta_{\text {lon }}\right)=K_{\text {eff }, \dot{V}} \cdot \begin{cases}15 \delta_{\text {lon }} & -1 \leq \delta_{\text {lon }}<0 \\ 85 \delta_{\text {lon }} & 0 \leq \delta_{\text {lon }} \leq 1\end{cases}
$$

Heading command filter The heading control filter has a sideslip $\beta$ control, direction command direction hold $(\mathrm{DCDH})$, and rate command direction hold (RCDH) mode. The sideslip $\beta$ control uses the derivative of the heading $\dot{\psi}$ in contrast to the yaw rate in body axes $r$ as in [17]. The sideslip controller is implemented as with the effectiveness $K_{\text {eff }, \beta}=\frac{\pi}{36}$

$$
\dot{\psi}_{\mathrm{c}}=-\left(K_{\mathrm{eff}, \beta} \delta_{\text {yaw }}-\beta\right)
$$

The other modes resemble the attitude command filter with $K_{\mathrm{eff}, \psi}=0.15$ and $K_{\mathrm{eff}, \psi}=2 \pi$ and the input $\delta_{\text {yaw }}$.

Coordinated turn The law for the coordinated turn is given as

$$
\ddot{\psi}_{\mathrm{c}}=K_{\mathrm{P}, \phi}\left(\frac{g \tan \dot{\phi}_{\mathrm{c}}}{V}-\dot{\psi}_{\mathrm{m}}\right)
$$

with $K_{\mathrm{P}, \phi}=2$, the gravitational acceleration $g$, and the total velocity $V$.

\section{Experiments and Results}

Simulation experiments are conducted to evaluate the proposed controller. Those experiments aim to demonstrate the controller's ability to perform a generic UAM mission, as shown in Figure 1], its stability and fulfillment of the requirements, as well as its performance by means of step responses. Selected experiments demonstrating the closed-loop system's main dynamics and capabilities are introduced subsequently.

TRC Experiment TRC can be used to precisely control the eVTOL's position over the ground. The vehicle starts in a trimmed hover state and gets a step command at $5 \mathrm{~s}$ from $0 \mathrm{~m}$ to $2 \mathrm{~m}$ on the position ${ }^{\mathrm{N}} \mathbf{x}_{\mathrm{y}}$ via the sidestick input $\delta_{\text {lat }}$. The result in Figure 5 shows the step response of a position step command. The step response has a rise time of $4.243 \mathrm{~s}$, a settling time of $17.599 \mathrm{~s}$, and an overshoot of $0.177 \%$. However, an undershoot of $7.88 \%$ is present. 


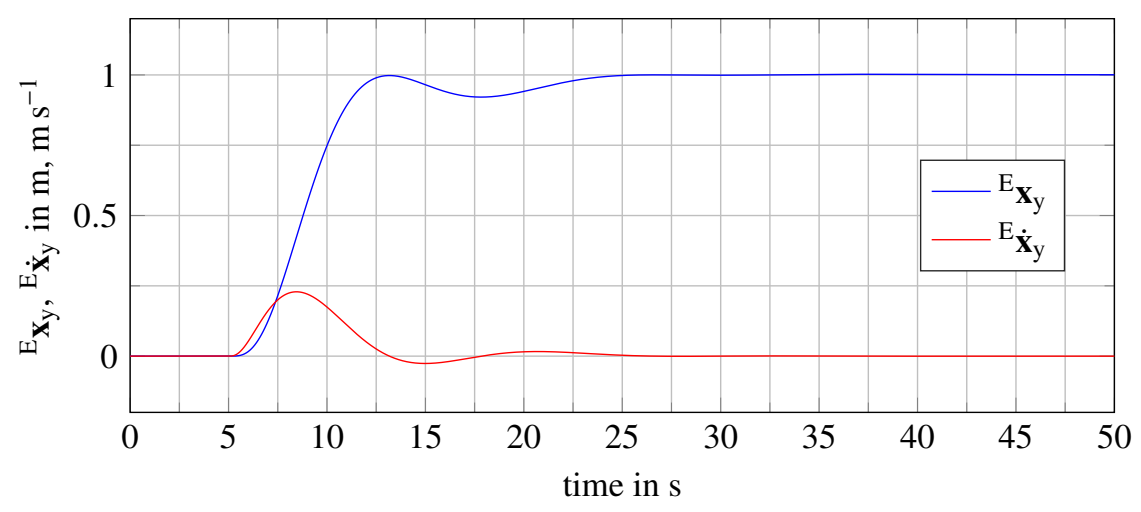

Fig. 5 Step response of basic position controller around TRC.

Transition Experiment The vehicle starts in a trimmed hover state. A step on the velocity ${ }^{\mathrm{B}} \mathbf{v}_{x}$ at $10 \mathrm{~s}$ from $0 \mathrm{~m} \mathrm{~s}^{-1}$ to $50 \mathrm{~m} \mathrm{~s}^{-1}$ is commanded. The feed-forward path is enabled, and the altitude and attitude are held during the experiment. Figure 6 shows the results of this experiment. Figure $6 \mathrm{a}$ exhibits the step response with a settling time of $50.484 \mathrm{~s}$, nearly no overshoot $\left(2 \times 10^{-5} \%\right)$, and a rise time of $39.996 \mathrm{~s}$. Figure 6 and Figure $6 \mathrm{~d}$ show the tilt angle and thrust trend, respectively. Those trends exhibit a smooth course during the transition and are similar to the trends from the trim study in [10]. The total thrust has a sharp trend at $60 \mathrm{~s}$, and the tilt angle at about $12 \mathrm{~s}$. Both trends reach a stable final value. The pitch angle in Figure 6 e is essentially maintained and stabilizes after a maximal deviation of $0.0047 \mathrm{rad}$.

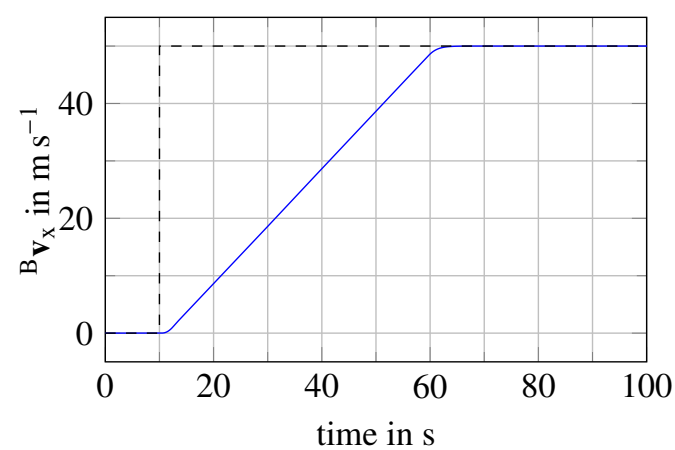

(a) Velocity over time with the commanded velocity - - -

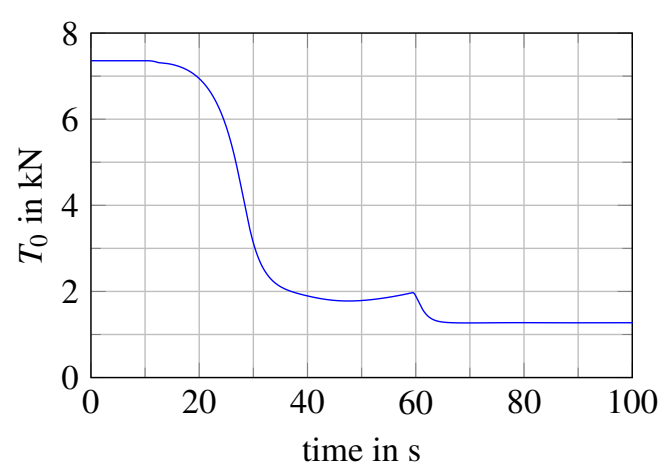

(c) Total thrust over time.

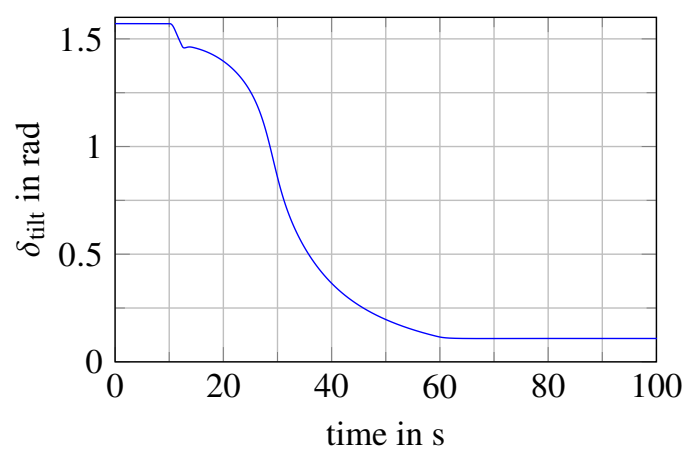

(b) Tilt angle over time.

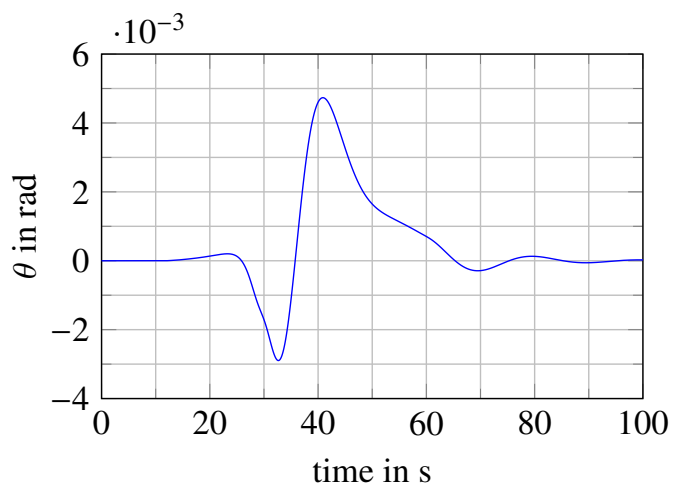

(d) Pitch angle over time.

Fig. 6 Acceleration from trimmed hover state to cruise flight at $50 \mathrm{~m} \mathrm{~s}^{-1}$. 
Complete Mission A complete mission, as shown in Figure1, is completed. The vehicle starts in a trimmed hover state at $h=0 \mathrm{~m}$, and gets height and velocity step commands sequentially. It climbs in hover mode, transits to cruise flight, climbs again during cruise, and vice versa. However, during the decline, the velocity and height are finally reduced in combination, offering a more efficient landing by reducing the pure hover time. The results shown in Figure 7 suggest that the controller can handle a generic UAM mission. Only a slight negative velocity $\left(-0.5 \mathrm{~m} \mathrm{~s}^{-1}\right)$ is reached during the initial climb maneuver, and the final velocity decline from $10 \mathrm{~m} \mathrm{~s}^{-1}$ approaches $0 \mathrm{~m} \mathrm{~s}^{-1}$ asymptotically.

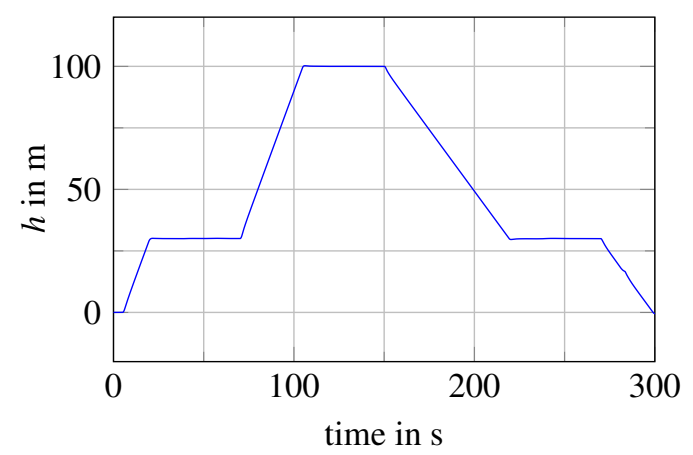

(a) Velocity over time

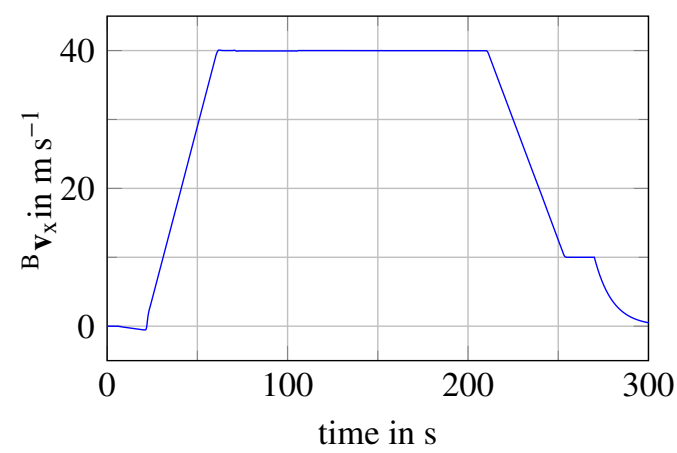

(b) Tilt angle over time.

Fig. 7 Compete flight profile of a generic UAM mission as defined in Figure 1 .

\section{Design Verification}

This section verifies the controller design by means of the requirements stated in Section III.B

Height response characteristics For the validation of the height response characteristics of the closed-loop system according to Section III.B, the vehicle starts in a trimmed hover state with no velocities and rotations. A step on the lever $\delta_{\text {lever }}$ in RCHH mode is commanded, i.e., a command on the height derivative $\dot{h}$. The step is executed at $5 \mathrm{~s}$ and has a final value of $1 \mathrm{~m} \mathrm{~s}^{-1}$.

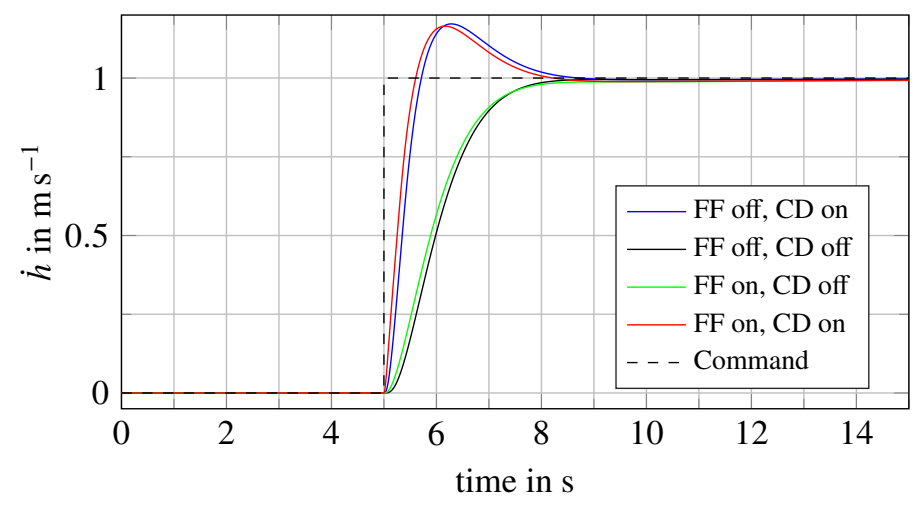

Fig. 8 Height rate step response with all combinations of enabled and disabled feed-forward paths.

As shown in Sections V.C and V.D the vertical velocity control loop has two essential structural design freedoms. Those are the feed-forward path in the linear controller $(F F)$, and the feed-forward path of the commanded height rate in the command filter $(C D)$. Combinations of both modes are tested to obtain the best setting. Figure 8 shows the results and characteristics of the different modes. The obtained step response characteristics are fitted into the first-order response, as described in Section III.B. The step response characteristics and received parameters are stated in Table 4.

Section III.B requires $T_{\mathrm{h}} \leq 5 \mathrm{~s}, \tau_{\mathrm{h}} \leq 0.2 \mathrm{~s}$, and a coefficient of determination of $0.97 \leq r^{2} \leq 1.03$. Only the combination of an enabled FF and a disabled CD fulfills the requirements. The corresponding linearized system around 
Table 4 Height rate step response characteristics.

\begin{tabular}{lcccc}
\hline Metric & FF off, CD on & FF off, CD off & FF on, CD off & FF on, CD on \\
\hline Rise time & $0.472 \mathrm{~s}$ & $1.625 \mathrm{~s}$ & $1.635 \mathrm{~s}$ & $0.427 \mathrm{~s}$ \\
Settling time & $3.019 \mathrm{~s}$ & $2.789 \mathrm{~s}$ & $2.825 \mathrm{~s}$ & $2.794 \mathrm{~s}$ \\
Overshoot & $17.428 \%$ & $0 \%$ & $0 \%$ & $16.981 \%$ \\
Fitted $T_{\mathrm{h}}$ & $0.275 \mathrm{~s}$ & $0.965 \mathrm{~s}$ & $0.919 \mathrm{~s}$ & $0.218 \mathrm{~s}$ \\
Fitted $\tau_{\mathrm{h}}$ & $0.079 \mathrm{~s}$ & $0.207 \mathrm{~s}$ & $0.164 \mathrm{~s}$ & $0.048 \mathrm{~s}$ \\
Fitting $r^{2}$ & 0.845 & 0.980 & 0.987 & 0.834 \\
\hline
\end{tabular}

the hover state yields a phase margin of $13.09 \mathrm{~dB}$ and a gain margin of $65^{\circ}$. Those values fulfill the requirements of $\geq 6 \mathrm{~dB}$ and $\geq 45^{\circ}$ with an additional margin.

Interaxis coupling In the pitch-roll-coupling experiment, shown in Figure 9 , the interaxis coupling of $\phi$ with $\theta$ is investigated. A step is commanded on the pitch axis during trimmed forward flight with ${ }^{\mathrm{B}} \mathbf{v}_{\mathrm{x}}=40 \mathrm{~m} \mathrm{~s}^{-1}$ and no other velocities or rotations. The effect on the roll (and yaw) axis is investigated. The results show that the closed-loop system fulfills the interaxis coupling requirements:

$$
\frac{\Delta \phi_{\mathrm{pk}}}{\Delta \theta_{4}} \approx \frac{-1.9446 \times 10^{-4} \mathrm{rad}}{0.0888 \mathrm{rad}} \approx 0.0022
$$

which is in the valid range $\left[-\frac{1}{4} ; \frac{1}{4}\right]$.

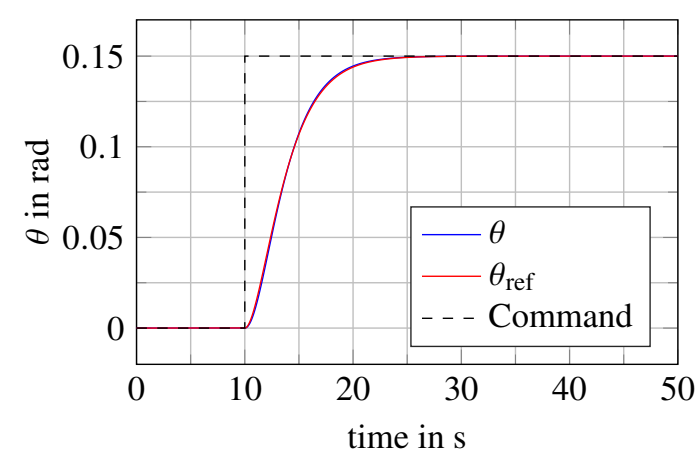

(a) Pitch angle trend over time.

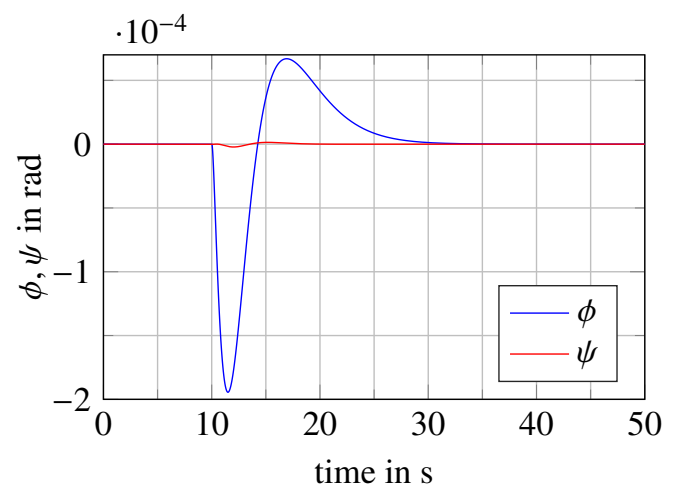

(b) Roll and yaw angle over time.

Fig. 9 Pitch step response showing the decoupling of the closed-loop system.

A similar experiment setup is applied to the roll and pitch coupling where the effect of a roll angle step of $0.2 \mathrm{rad}$ during trimmed forward flight with a velocity of $40 \mathrm{~m} \mathrm{~s}^{-1}$ is investigated. The influence of this step on the pitch angle specified in the interaxis coupling requirements in Section III.B is fulfilled as well:

$$
\frac{\Delta \theta_{\mathrm{pk}}}{\Delta \phi} \approx \frac{0.0114 \mathrm{rad}}{0.1981 \mathrm{rad}} \approx 0.0576
$$

which is in the valid range $\left[-\frac{1}{4} ; \frac{1}{4}\right]$.

The height response experiment shown in Figure 8 investigates the height derivative step response of the closed-loop system. Interaxis coupling of the vertical acceleration and the pitch angle can be drawn from this experiment. The corresponding interaxis coupling requirement is fulfilled by the enabled FF and disabled CD controller:

$$
\frac{\Delta \theta_{\mathrm{pk}}}{\Delta n_{\mathrm{zk}}} \approx \frac{2.7061 \times 10^{-5 \circ}}{-0.7101 \mathrm{~m} \mathrm{~s}^{-2}} \approx-3.8106 \times 10^{-5} \circ \mathrm{s}^{2} \mathrm{~m}^{-1}<0.0573^{\circ} \mathrm{s}^{2} \mathrm{~m}^{-1}
$$




\section{Discussion}

Sections VI and VII show several experiments that support the hypothesis of a tilt-wing eVTOL controller for a uniform control system. These results are discussed subsequently.

\section{A. Experiments}

TRC Experiment The TRC mode can be used to control the eVTOL's position over the ground precisely, e.g., during take-off and landing. The closed-loop system fulfills the handling quality requirements specified in Section III.B. The undershoot in Figure 5 is caused by the integrator's slow dynamics in the TRC's PI controller. An integrator-less controller may not exhibit any noticeable undershoot but the same overshoot. The integrator integrates the overshoot's error and compensates for this error through an undershoot. However, the integrator in the PI controller is crucial in this situation since it trims the vehicle to its new state. The kinematic inversion in the TRC does not consider aerodynamic effects, i.e., mainly the drag force due to the sidewards motion. This effect depends on multiple parameters and is complex to model. Thus, the integrator compensates for this inaccuracy by maintaining a static roll or pitch angle command counteracting these effects. Possible ways to overcome the issue of the present undershoot are an improvement of the inversion or an alternative linear controller. Implementing certain additional dynamics may be hard since the tilt-wing aerodynamics are not fully understood yet [10]. An alternative solution is to use a Total Energy Control System [32] (TECS)-like linear controller. Thus, the original TRC law stated in (11) may be replaced by the following law

$$
{ }^{\mathrm{N}} \mathbf{v}_{\mathrm{xy}, \mathrm{c}}=-K_{\mathrm{P}}{ }^{\mathrm{N}} \mathbf{v}_{\mathrm{xy}, \mathrm{m}}+\frac{K_{\mathrm{I}}}{s}\left(\frac{1}{T_{\mathrm{TRC}} s+1}{ }^{\mathrm{N}} \mathbf{v}_{\mathrm{xy}, \mathrm{d}}-{ }^{\mathrm{N}} \mathbf{v}_{\mathrm{xy}, \mathrm{m}}\right)+K_{\mathrm{FF}} \frac{s}{T_{\mathrm{TRC}} s+1}{ }^{\mathrm{N}} \mathbf{v}_{\mathrm{xy}, \mathrm{d}}
$$

This issue will be focused in future research.

Transition Experiment The experiment is a first step towards the complete mission experiment and shows that the eVTOL can transit to the final velocity of $50 \mathrm{~m} \mathrm{~s}^{-1}$ smoothly. Thus, it is capable of transitioning from hover or thrust-born to forward or wing-borne flight. The altitude is maintained constant during the experiment. Handling the transition well while holding its altitude is a crucial part of tilt-wing aircraft. Furthermore, the tilt angle and total thrust trend are similar to the trends in the trim study in [10]. This suggests a smooth transition along a trimmed trajectory. The edge in the total thrust trend at $60 \mathrm{~s}$ and the edge in the tilt angle trend at $12 \mathrm{~s}$ are present because at these points, the acceleration changes sharply. The closed-loop system cannot handle this behavior smoothly. However, the trends are still in the possible envelope of the actuators. Optimizing and introducing more precise envelope limits on the acceleration command may solve this issue.

Complete Mission The results in Figure 7 suggest that the closed-loop system is able to complete a generic UAM mission as stated in Figure 1 smoothly. The transition behaves similarly to Figure 6 except for a slight negative velocity during climb. Furthermore, during the final deceleration phase, the vehicle approaches ${ }^{\mathrm{B}} \mathbf{v}_{\mathrm{x}}=0 \mathrm{~m} \mathrm{~s}^{-1}$ asymptotically. This is caused by the limitation of the tilt angle to at most $90^{\circ}$. Thus, no reverse thrust and acceleration are possible in the hover mode. Solely the drag force, which depends on the current velocity, is utilized for stopping the vehicle. Real-world eVTOLs thus may decelerate faster by tilting $>90^{\circ}$ or by pitching the body up slightly to achieve the same effect.

\section{B. Design Verification}

During the design verification, the fulfillment of the requirements from Section III.B could be shown. A suitable control parameter ensuring the height response characteristics is selected. In the case of an enabled feed-forward path in the linear height controller combined with a disabled feed-forward path in the altitude command filter, the requirements are fulfilled. Section VII shows this argumentation in more detail.

The interaxis coupling requirements from roll to pitch and pitch to roll are fulfilled. This is shown in SectionVII and exemplified for the pitch-roll-coupling in Figure 9

\section{Overall Outcome}

Several experiments and design verifications suggest that the system, and thus the inner-loop controller, exhibits a stable behavior in all scenarios and fulfills the specified requirements. The vehicle is able to manage every part of a generic UAM mission. This suggests that the closed-loop system is suitable for UAM scenarios. The vehicle handles 
the complex transition well. However, the aerodynamics in the used model are simplified and may not include possible little-studied malign effects. The system decouples the axes well in all experiments. Thus, the realization of a unified control framework around the inner-loop controller is possible.

Possible and precise maneuvering in hover mode can be handled with TRC. The transition experiment shows the capability to transit smoothly from thrust-borne hover flight to wing-born cruise flight. Subsequently, it is shown that the controller can control the tilt-wing eVTOL over the complete mission profile, as shown in Figure 1 A height change during cruise flight is possible in a smooth way, according to the height response design verification experiment.

The controller is designed to fulfill selected handling quality and flight control requirements. Section[VII shows the compliance of the controller with those requirements. The controller can decouple the different axes. In accordance with the ADS33E-PRF standard, the controller shows appropriate interaxis coupling behavior, i.e., a sufficient decoupling. Independently controlling each axis is thus possible with the designed controller.

Furthermore, the controller has integrated envelope protection, ensuring the vehicle stays within its comfort limits. In summary, it is possible to fly a complete mission without the need for complex inceptor coordination. Other inceptor-command mappings can easily be implemented without changing the inner-loop controller due to the decoupling capabilities. Thus, other suitable and future command concepts can be established quickly. The implemented inceptor mapping is one possible construct.

The present work is mainly limited by the dynamic model from [10]. [41] states that neglecting the gyroscopic effects from tilting rotors, as well as the changing center of gravity, has a notable influence on dynamic behaviors. However, this work focuses on providing a proof of concept. Future studies will consider the neglected effects.

\section{Conclusion and Outlook}

This paper shows the design of an INDI controller for an integrated tilt-wing eVTOL model to provide the basis for a future unified control system. A proof-of-concept for the unified control scheme is provided. Relevant requirements for the flight control design are identified and an inner-loop INDI controller according to those is designed. Furthermore, multiple outer-loop controllers for realizing the requirements are implemented and tuned. The total closed-loop system is evaluated by means of the requirements as well as a generic UAM mission. The results suggest that the developed control system fulfills the stated requirements and can control the eVTOL sufficiently. The dynamic model and the control system can be used as a basis for future research, especially for developing a unified control framework. The proof of concept for a unified control framework for a tilt-wing eVTOL vehicle is provided. Although the control law is designed to be generic and robust, only one particular tilt-wing eVTOL configuration is investigated in this work's scope. A transfer of the obtained knowledge to other eVTOL or aircraft configurations is desirable and is favored by the nature of inversion-based control laws. Furthermore, many adverse effects, e.g., wind turbulence and failures, are neglected.

Future research may look into these open points in more detail and investigate, e.g., passenger comfort, robustness, and disturbance rejection. Further research may also focus on more details of the control system and the dynamic model. It may include a more detailed model of the electric motor and aerodynamics as suggested by [10]. Furthermore, the development of a unified control scheme for tilt-wing eVTOLs is another crucial next step. Handling qualities will also be investigated via human-in-the-loop tests [42, 43]. This future development heavily benefits from the proposed controller.

\section{References}

[1] Vieira, D. R., Silva, D., and Bravo, A., "Electric VTOL aircraft: the future of urban air mobility (background, advantages and challenges)," International Journal of Sustainable Aviation, Vol. 5, No. 2, 2019, p. 101. https://doi.org/10.1504/ijsa.2019.101746

[2] Bacchini, A., and Cestino, E., "Electric VTOL Configurations Comparison," Aerospace, Vol. 6, No. 3, 2019 , p. 26. https://doi.org/10.3390/aerospace6030026

[3] Lombaerts, T., Kaneshige, J., and Feary, M., "Control Concepts for Simplified Vehicle Operations of a Quadrotor eVTOL Vehicle," AIAA Aviation 2020 Forum, 2020.

[4] Hartmann, P., Meyer, C., and Moormann, D., "Unified Velocity Control and Flight State Transition of Unmanned Tilt-Wing Aircraft," Journal of Guidance, Control, and Dynamics, Vol. 40, No. 6, 2017, pp. 1348-1359. https://doi.org/10.2514/1.g002168

[5] Lovering, Z., "Vahana Configuration Trade Study - Part I," , dec 2016. URL https://acubed.airbus.com/blog/vahana/vahanaconfiguration-trade-study-part-i. accessed: 2020-11-17. 
[6] Bower, G., "Vahana Configuration Trade Study - Part II," , feb 2017. URL https://acubed.airbus.com/blog/vahana/vahanaconfiguration-trade-study-part-ii accessed: 2020-11-17.

[7] Droandi, G., Syal, M., and Bower, G., "Tiltwing Multi-Rotor Aerodynamic Modeling in Hover, Transition and Cruise Flight Conditions," 2018.

[8] Pradeep, P., and Wei, P., "Energy Optimal Speed Profile for Arrival of Tandem Tilt-Wing eVTOL Aircraft with RTA Constraint," 2018.

[9] Montagnani, D., Tugnoli, M., Zanotti, A., Syal, M., and Droandi, G., "Analysis of the Interactional Aerodynamics of the Vahana eVTOL Using a Medium Fidelity Open Source Tool," VFS Aeromechanics for Advanced Vertical Flight Technical Meeting, 2020.

[10] May, M., Milz, D., and Looye, G., "Dynamic Modeling and Analysis of Tilt-Wing Electric Vertical Take-Off and Landing Vehicles," AIAA SCITECH 2022 Forum, 2022. https://doi.org/10.2514/6.2022-0263

[11] Chauhan, S. S., and Martins, J. R. R. A., “Tilt-Wing eVTOL Takeoff Trajectory Optimization,” Journal of Aircraft, Vol. 57, No. 1, 2020, pp. 93-112. https://doi.org/10.2514/1.C035476.

[12] Cetinsoy, E., Dikyar, S., Hancer, C., Oner, K., Sirimoglu, E., Unel, M., and Aksit, M., "Design and construction of a novel quad tilt-wing UAV," Mechatronics, Vol. 22, No. 6, 2012, pp. 723-745. https://doi.org/10.1016/j.mechatronics.2012.03.003

[13] Yanguo, S., and Huanjin, W., "Design of Flight Control System for a Small Unmanned Tilt Rotor Aircraft," Chinese Journal of Aeronautics, Vol. 22, No. 3, 2009, pp. 250 - 256. https://doi.org/10.1016/S1000-9361(08)60095-3.

[14] Denham, J., and Paines, J., "Converging on a Precision Hover Control Strategy for the F-35B STOVL Aircraft," AIAA Guidance, Navigation and Control Conference and Exhibit, 2008. https://doi.org/10.2514/6.2008-6331

[15] Nordeen, L., Harrier II : validating V/STOL, Naval Institute Press, Annapolis, Md, 2006.

[16] Lombaerts, T., Kaneshige, J., Schuet, S., Hardy, G., Aponso, B. L., and Shish, K. H., Nonlinear Dynamic Inversion Based Attitude Control for a hovering quad tiltrotor eVTOL vehicle, 2019. https://doi.org/10.2514/6.2019-0134

[17] Lombaerts, T., Kaneshige, J., Schuet, S., Aponso, B. L., Shish, K. H., and Hardy, G., "Dynamic Inversion based Full Envelope Flight Control for an eVTOL Vehicle using a Unified Framework," AIAA Scitech 2020 Forum, 2020. https: //doi.org/10.2514/6.2020-1619

[18] Di Francesco, G., and Mattei, M., "Modeling and Incremental Nonlinear Dynamic Inversion Control of a Novel Unmanned Tiltrotor," Journal of Aircraft, Vol. 53, No. 1, 2016, pp. 73-86. https://doi.org/10.2514/1.C033183

[19] Hartmann, P., Schütt, M., and Moormann, D., "Konzept eines stetigen Bahnreglers für den vollständigen Flugbereich eines Kippflügelflugzeuges," Publikationen zum DLRK 2014 : 63. Deutscher Luft- und Raumfahrtkongress 2014, edited by DGLR, 2014.

[20] Cetinsoy, E., Hancer, C., Oner, K. T., Sirimoglu, E., and Unel, M., "Aerodynamic Design and Characterization of a Quad Tilt-Wing UAV via Wind Tunnel Tests," Journal of Aerospace Engineering, Vol. 25, No. 4, 2012, pp. 574-587. https://doi.org/10.1061/(asce)as.1943-5525.0000161

[21] Öner, K. T., Çetinsoy, E., SIRIMOĞLU, E., Hançer, C., Ünel, M., Akşit, M. F., Gülez, K., and Kandemir, I., "Mathematical modeling and vertical flight control of a tilt-wing UAV," Turkish Journal of Electrical Engineering \& Computer Sciences, Vol. 20, No. 1, 2012, pp. 149-157.

[22] Hong, G., Wei, T., and Ding, X., "Multi-Objective Optimal Design of Permanent Magnet Synchronous Motor for High Efficiency and High Dynamic Performance," IEEE Access, Vol. 6, 2018, pp. 23568-23581. https://doi.org/10.1109/ACCESS.2018.2828802

[23] Ciuryla, M., Liu, Y., Farnsworth, J., Kwan, C., and Amitay, M., "Flight Control Using Synthetic Jets on a Cessna 182 Model," Journal of Aircraft, Vol. 44, No. 2, 2007, pp. 642-653. https://doi.org/10.2514/1.24961

[24] Champine, R. A., Kelley, H., and Reeder, J. P., "NASA TM X-1914: Summary of a flight-test evaluation of the CL-84 tilt-wing V/STOL aircraft,” Tech. rep., NASA Langley Research Center, 1970.

[25] Stewart, E., "A piloted simulation study of advanced controls and displays for novice general aviation pilots," 32nd Aerospace Sciences Meeting and Exhibit, 1994. https://doi.org/10.2514/6.1994-276. 
[26] Walter, A., McKay, M., Niemiec, R., Gandhi, F., and Ivler, C., "Handling Qualities Based Assessment of Scalability for Variable-RPM Electric Multi-Rotor Aircraft," Proceedings of the 75th Vertical Flight Society Annual Forum, Philadelphia, Pennsylvania, 2019.

[27] Samuel Crews, William Smith, Barry Baskett, and Dr. Larry Daniel, "ADS33E-PRF: Aeronautical design standard performance specification handling qualitiew requirements for military rotorcraft," Tech. rep., United States Army Aviation and Missile Command, 2000.

[28] "AS94900A: Vehicle Management Systems - Flight Control Function, Design, Installation and Test of Piloted Military Aircraft, General Specification For,”, 2018. https://doi.org/10.4271/as94900a

[29] “MIL-HDBK-1797: Flying Qualities of Piloted Aircraft,” Tech. rep., US Department of Defense, 1997.

[30] Padfield, G., and Meyer, M., "Progress in Civil Tilt-Rotor Handling Qualities," Journal of The American Helicopter Society - J AMER HELICOPTER SOC, Vol. 51, 2006. https://doi.org/10.4050/1.3092880

[31] Elevate, U., "Uber Air Vehicle Requirements and Missions," , 2019. URL https://s3.amazonaws.com/uber-static/elevate/ Summary+Mission+and+Requirements.pdf accessed: 2020-11-17.

[32] Looye, G., “An Integrated Approach to Aircraft Modelling and Flight Control Law Design,” Ph.D. thesis, Universität Delft, NL, Januar 2008.

[33] Simplício, P., Pavel, M., van Kampen, E., and Chu, Q. P., "An Acceleration Measurements-based Approach for Helicopter Nonlinear Flight Control using Incremental Nonlinear Dynamic Inversion,” Control Engineering Practice, Vol. 21, No. 8, 2013, pp. 1065-1077.

[34] Smeur, E. J. J., Chu, Q., and de Croon, G. C. H. E., "Adaptive Incremental Nonlinear Dynamic Inversion for Attitude Control of Micro Air Vehicles," Journal of Guidance, Control, and Dynamics, Vol. 39, No. 3, 2016, pp. 450-461. https://doi.org/10.2514/1.g001490

[35] Sieberling, S., Chu, Q. P., and Mulder, J. A., "Robust Flight Control Using Incremental Nonlinear Dynamic Inversion and Angular Acceleration Prediction," Journal of Guidance, Control, and Dynamics, Vol. 33, No. 6, 2010 , pp. $1732-1742$. https://doi.org/10.2514/1.49978

[36] Acquatella, P., Falkena, W., van Kampen, E.-J., and Chu, Q. P., "Robust Nonlinear Spacecraft Attitude Control using Incremental Nonlinear Dynamic Inversion.” AIAA Guidance, Navigation, and Control Conference, 2012. https://doi.org/10.2514/6.20124623

[37] Grondman, F., Looye, G., Kuchar, R. O., Chu, Q. P., and Kampen, E.-J. V., "Design and Flight Testing of Incremental Nonlinear Dynamic Inversion-based Control Laws for a Passenger Aircraft," 2018 AIAA Guidance, Navigation, and Control Conference, 2018. https://doi.org/10.2514/6.2018-0385

[38] Wang, X., van Kampen, E.-J., Chu, Q., and Lu, P., "Stability Analysis for Incremental Nonlinear Dynamic Inversion Control," Journal of Guidance, Control, and Dynamics, Vol. 42, No. 5, 2019, pp. 1116-1129. https://doi.org/10.2514/1.G003791

[39] Looye, G., "Dynamic Inversion: an incrementally evolving methodology for flight control design,” AIAA, 2022. To be published.

[40] Matamoros, I., and de Visser, C. C., "Incremental Nonlinear Control Allocation for a Tailless Aircraft with Innovative Control Effectors,” 2018 AIAA Guidance, Navigation, and Control Conference, 2018. https://doi.org/10.2514/6.2018-1116.

[41] Haixu, L., Xiangju, Q., and Weijun, W., "Multi-body Motion Modeling and Simulation for Tilt Rotor Aircraft," Chinese Journal of Aeronautics, Vol. 23, No. 4, 2010, pp. 415-422. https://doi.org/10.1016/S1000-9361(09)60236-3

[42] Seefried, A., Pollok, A., Kuchar, R., Hellerer, M., Leitner, M., Milz, D., Schallert, C., Kier, T., Looye, G., and Bellmann, T., "Multi-Domain Flight Simulation with the DLR Robotic Motion Simulator," 2019 Spring Simulation Conference, 2019. https://doi.org/10.22360/springsim.2019.anss.011

[43] Milz, D., Weiser, C., van der Linden, F., Hellerer, M., Seefried, A., and Bellmann, T., "Advances in Flight Dynamics Modeling and Flight Control Design by Using the DLR Flight Visualization and Flight Instruments Libraries," 13th International Modelica Conference, Linköping Electronic Conference Proceedings, Vol. 157, Linköping University Electronic Press, Linköpings universitet, 2019, pp. 481-488. https://doi.org/10.3384/ecp19157481. 